\title{
Article \\ A Clot Waveform Analysis Showing a Hypercoagulable State in Patients with Malignant Neoplasms
}

\author{
Mayu Kobayashi ${ }^{1}$, Hideo Wada ${ }^{2, *}$ C, Shunsuke Fukui ${ }^{1}$, Hiroki Mizutani ${ }^{1}$, Yuhuko Ichikawa ${ }^{3}$, Katsuya Shiraki ${ }^{2}$, \\ Isao Moritani ${ }^{1}$, Hidekazu Inoue ${ }^{1}$, Motomu Shimaoka ${ }^{4}$ and Hideto Shimpo ${ }^{5}$ \\ 1 Mie Prefectural General Medical Center, Department of Gastroenterology, Yokkaichi 510-0885, Japan; \\ xsvaria1010@gmail.com (M.K.); shunsuke-fukui@mie-gmc.jp (S.F.); n279828658b79ku@gmail.com (H.M.); \\ isao-moritani@mie-gmc.jp (I.M.); hidekazu-inoue@mie-gmc.jp (H.I.) \\ 2 Mie Prefectural General Medical Center, Department of General and Laboratory Medicine, \\ Yokkaichi 510-0885, Japan; katsuya-shiraki@mie-gmc.jp \\ 3 Mie Prefectural General Medical Center, Department of Central Laboratory Medicine, \\ Yokkaichi 510-0885, Japan; ichi911239@yahoo.co.jp \\ 4 Department of Molecular Pathobiology and Cell Adhesion Biology, Mie University Graduate School of \\ Medicine, Tsu 514-8507, Japan; motomushimaoka@gmail.com \\ 5 Mie Prefectural General Medical Center, Yokkaichi 510-0885, Japan; hideto-shimpo@mie-gmc.jp \\ * Correspondence: wadahide@clin.medic.mie-u.ac.jp; Tel.: +81-59-345-2321
}

check for updates

Citation: Kobayashi, M.; Wada, H.; Fukui, S.; Mizutani, H.; Ichikawa, Y.; Shiraki, K.; Moritani, I.; Inoue, H.; Shimaoka, M.; Shimpo, H. A Clot Waveform Analysis Showing a Hypercoagulable State in Patients with Malignant Neoplasms. J. Clin. Med. 2021, 10, 5352. https://doi.org/ $10.3390 /$ jcm10225352

Academic Editor: Massimo Morfini

Received: 14 October 2021

Accepted: 15 November 2021

Published: 17 November 2021

Publisher's Note: MDPI stays neutral with regard to jurisdictional claims in published maps and institutional affiliations.

Copyright: (C) 2021 by the authors Licensee MDPI, Basel, Switzerland. This article is an open access article distributed under the terms and conditions of the Creative Commons Attribution (CC BY) license (https:// creativecommons.org/licenses/by/ $4.0 /)$.

\begin{abstract}
Objective: hypercoagulability in patients with malignant neoplasm were evaluated to examine the relationship with thrombosis. (2) Methods: clot waveform analysis (CWA) - activated partial thromboplastin time (APTT) and CWA—small amount of tissue factor induced FIX activation (sTF/FIXa) assays were performed in 92 patients with malignant neoplasm and the relationship between hypercoagulability and thrombosis was retrospectively examined. (3) Results: The study population included 92 patients with malignant neoplasms. Twenty-six (28.3\%) had thrombotic diseases and $9(9.8 \%)$ patients died within 28 days after the CWA. The peak time of the CWA-APTT could not show hypercoagulability in patients with malignant neoplasms. There were almost no significant differences in the peak times of the sTF/FIXa among patients with malignant neoplasms and healthy volunteers. In contrast, the peak heights of the CWA-sTF/FIXa in patients with various malignant neoplasms were significantly higher than those in healthy volunteers. Furthermore, among patients with malignant neoplasms, the peak heights of the sTF/FIXa in patients with thrombosis were significantly higher than those in patients without thrombosis. (4) Conclusions: although the routine APTT cannot evaluate the hypercoagulability, the peak heights of CWA-sTF/FIXa were significantly high in patients with malignant neoplasms, especially in those with thrombosis, suggesting that an elevated peak height of the CWA-sTF/FIXa may be a risk factor for thrombosis.
\end{abstract}

Keywords: CWA; APTT; sTF/FIXa; cancer; thrombosis

\section{Introduction}

Cancer associated thrombosis [1,2] has been recently attracted attention by many physicians and researchers. The incidence of venous thromboembolism in patients with cancer is $0.5 \%$, while that in the general population is $0.1 \%$ and active cancer accounts for $20 \%$ of the overall incidence of venous thromboembolism [3]. Thrombosis is a leading cause of noncancer death in cancer patients and includes both arterial and venous thromboembolism [4]. Although venous thromboembolism is considered one of the most important thrombotic diseases in cancer patients [1,5], Trousseau syndrome [6] is well-known thrombotic syndrome in cancer patients. This syndrome is first noted that unexpected or migratory thrombophlebitis could be a forewarning of an occult visceral malignancy [7]. Thereafter, this syndrome is extended as cancer-associated thrombosis, including acute cerebral infarction, venous thromboembolism and chronic disseminated intravascular coagulation (DIC) 
due to hypercoagulability [6,8-11]. Cancer-associated thrombosis is a heterogenous disease and various factors, including leukocytosis, thrombocytosis, several physical factors, and increased coagulation factors have been proposed as risk factors for thrombosis [1]. Hypercoagulability, including increased tissue factor (TF), which activates the extrinsic pathway of the coagulation system and TF-positive microparticles, was proposed as a risk factor for cancer-associated thrombosis [1]. Activation of the coagulation cascade through tumor growth and metastasis may cause thrombosis in cancer [12]. Platelet activation is also proposed as the mechanism of cancer-associated thrombosis [13]. Therefore, an early diagnosis and prophylaxis for thrombosis may be important in patients with cancer [6].

Although elevated D-dimer is useful for detecting thrombosis in patients with cancer $[14,15]$, there are no routine test for hypercoagulability. Recently, a clot waveform analysis (CWA) to activated partial thromboplastin time (APTT) has been developed to analyze to evaluate hemostatic abnormalities in patients with bleeding tendency and to monitor anticoagulant therapy $[16,17]$. Furthermore, the use of the small amount of TF induced FIX activation assay (sTF/FIXa) assay can evaluate the hemostatic abnormalities including platelet abnormalities [18].

In this study, hypercoagulability in patients with malignant neoplasms was investigated using the CWA-APTT and STF/FIXa and the relationship between the results of these tests and thrombosis was examined.

\section{Materials and Methods}

The study population included and 92 patients with the following conditions who were managed at Mie Prefectural General Medical Center from 21 August 2020 to 20 August 2021; hepatocellular carcinoma $(n=23)$; colon cancer $(n=4)$; stomach cancer $(n=8)$; prostate cancer $(n=5)$; biliary tract cancer $(n=7)$; lung cancer $(n=6)$; pancreatic cancer $(n=10)$; esophageal cancer $(n=4)$; malignant lymphoma $(n=14)$; myelodysplastic syndrome $(\mathrm{n}=10)$; other cancer $(\mathrm{n}=5$; origin unknown, $\mathrm{n}=2$ melanoma, $\mathrm{n}=1$; uterus cancer, $\mathrm{n}=1$ and ovarian cancer, $\mathrm{n}=1)$, including hepatocellular carcinoma and colon cancer $(\mathrm{n}=1)$; stomach and prostate cancer $(\mathrm{n}=1)$; stomach and biliary tract cancer $(\mathrm{n}=1)$, stomach and lung cancer $(\mathrm{n}=1)$. The study involved 18 healthy volunteers ( 8 males and 12 females; 21 to 56 years old).

DIC was diagnosed using the Japanese Ministry of Health Labor and Welfare criteria for DIC [19]. Acute cerebral infarction was diagnosed using computed tomography or magnetic resonance imaging, acute coronary syndrome was diagnosed using coronary angiography, electrocardiography and was based on elevated troponin levels, venous thromboembolism was diagnosed using computed tomography or venous Doppler ultrasound. In the CWA-APTT and STF/FIXa, 20 healthy volunteers (12 females and 8 males; median age, 35.5 years old; $25-75$ percentile, 33.0-35.0 years old) were also examined as control.

The study protocol (2019-K9) was approved by the Human Ethics Review Committee of Mie Prefectural General Medical Center, and informed consent was obtained from each participant. This study was carried out in accordance with the principles of the Declaration of Helsinki.

The CWA-APTT was performed using APTT-SP ${ }^{\circledR}$, which uses silica as an activator of FXII and synthetic PLs (Instrumentation Laboratory; Bedford, MA, USA) and platelet poor plasma, with an ACL-TOP ${ }^{\circledR}$ system (Instrumentation Laboratory) as previously reported $[16,20]$. The CWA-sTF/FIXa assay was performed using platelet rich plasma and 2000-fold diluted HemosIL RecombiPlasTin 2G (TF concentration < 0.1 pg $/ \mathrm{mL}$; Instrumentation Laboratory). Three curves are shown on the monitor of this system [11]. One curve shows the changes in the absorbance observed while measuring the APTT, corresponding to fibrin formation (FF, navy line). The second (first derivative peak, 1st DP; red line), corresponds to the coagulation velocity, and the third (second derivative peak, 2nd DP; light blue) corresponds to the coagulation acceleration. The height and time of the 1st DP, 2nd DP and FF are called the 1st DP height (1st DPH) and 1st DP time (1st DPT), 
2nd DPH and 2nd DPT, and FFH and FFT, respectively (Figure 1). Platelet rich plasma was prepared by centrifugation at $900 \mathrm{rpm}$ for $15 \mathrm{~min}$ (platelet count, $40 \times 10^{10} / \mathrm{L}$ ), and platelet-poor plasma was prepared by centrifugation at $3000 \mathrm{rpm}$ for $15 \mathrm{~min}$ (platelet count, $\left.<0.5 \times 10^{10} / \mathrm{L}\right)[18]$.
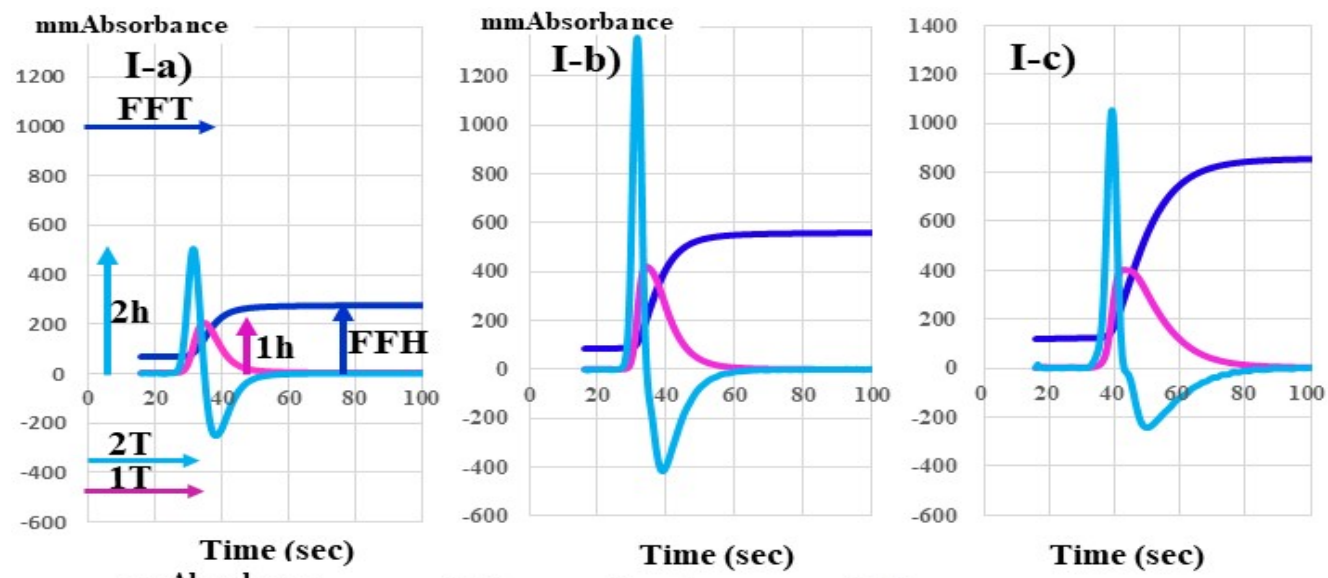

Time (sec)

Time (sec)

mmAbsorbance $\longrightarrow 250 ' 1400$

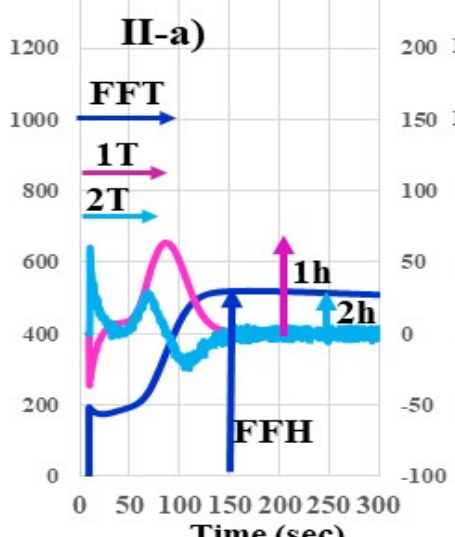

Time (sec)
II-b)

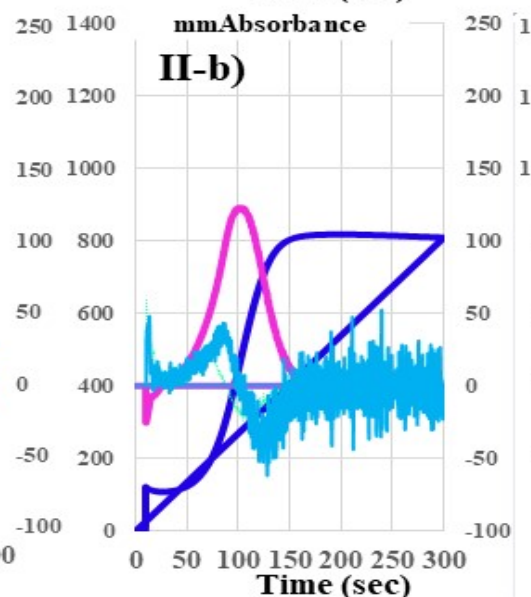

Time (sec)

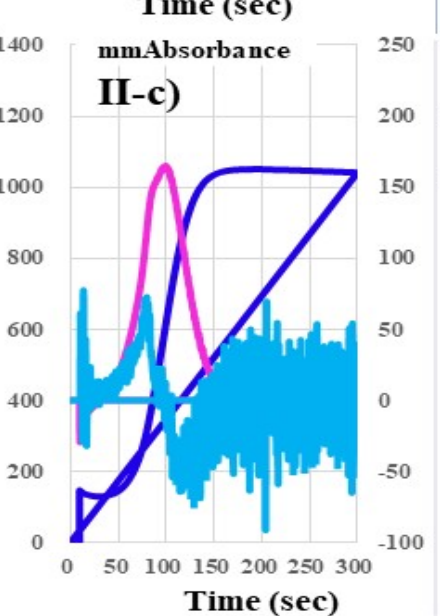

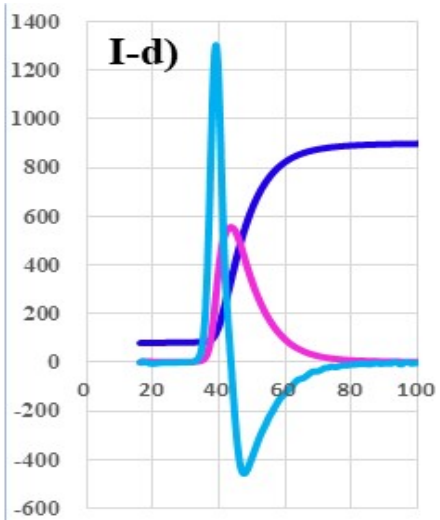

Time (sec)
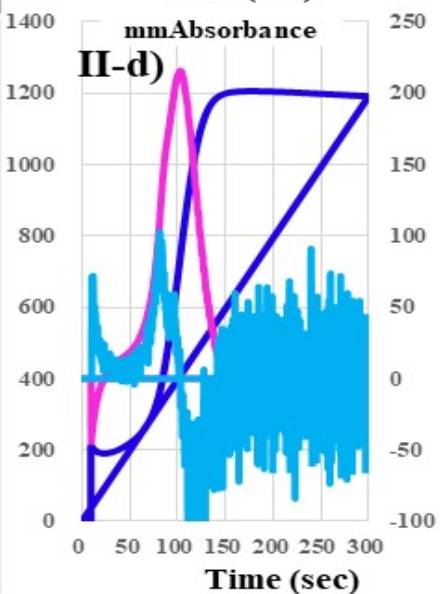

Figure 1. CWA-APTT (I) and CWA-sTF-FIXa (II) in a healthy volunteer (a), pancreatic cancer patient without thrombosis (b), pancreatic cancer patient before ACI (c) and patient with ACI (d). APTT, activated partial thromboplastin time; sTF/FIXa, small tissue factor induced FIX activation assay; ACI, acute cerebral thrombosis; navy line, fibrin formation curve; red line, 1st derivative curve (velocity); light-blue line, 2nd derivative curve (acceleration); 1T, 1st derivative peak time; 2T, 2nd derivative peak time; FFT, fibrin formation time; $1 \mathrm{~h}$, 1st derivative peak height; $2 \mathrm{~h}$, 2nd derivative peak height; FFH, fibrin formation height, TM (mm abs); transmittance ( $\mathrm{mm}$ absorbance).

\section{Statistical Analyses}

The data are expressed as the median (range). The significance of differences between groups was examined using the Mann-Whitney U-test. $p$ values of $<0.05$ were considered to indicate a statistical significance. All statistical analyses were performed using the Stat-Flex software program (version 6; Artec Co Ltd., Osaka, Japan).

\section{Results}

Figure 1 shows that there were no significant differences in the peak time of the APTT among a healthy volunteer, pancreatic cancer patient without and with acute cerebral infarction and non-cancer patient with acute cerebral infarction. Although the peak heights of the CWA-APTT were significantly higher in these three patients than healthy volunteer, there was no significant difference in the peak heights of the APTT among the above three patients. The CWA-sTF/FIXa assay shows that the peak heights were markedly higher in pancreatic cancer patient with acute cerebral infarction than in that without, whose levels were higher than those of healthy volunteer. Table 1 shows 92 patients with malignant 
neoplasms, $26(28.3 \%)$ of whom had associated thrombotic diseases (12 peripheral artery thrombosis and venous thromboembolism, $\mathrm{n}=12$; acute cerebral infarction, $\mathrm{n}=7$; acute coronary syndrome, $\mathrm{n}=4$; DIC, $\mathrm{n}=2$; and thrombotic microangiopathy, $\mathrm{n}=1$ ). Peripheral artery thrombosis and venous thromboembolism included deep vein thrombosis $(n=5)$, portal vein thrombosis $(n=4)$ and pulmonary.

Table 1. Subjects.

\begin{tabular}{cccccccccccc}
\hline Disease & N & Age & Male & PAVTE & ACI & ACS & DIC & TMA & Mortality \\
\hline Hepatocellular carcinoma $^{*}$ & 23 & $73.4 \pm 6.9$ & 18 & 3 & 2 & 1 & 0 & 0 & $0(0 \%)$ \\
\hline Colon cancer $^{*}$ & 4 & $74.8 \pm 11.2$ & 3 & 1 & 0 & 0 & 0 & 0 & $0(0 \%)$ \\
\hline Stomach cancer $^{\#, \&,+}$ & 8 & $76.6 \pm 9.6$ & 8 & 0 & 1 & 0 & 1 & 1 & $3(37.5 \%)$ \\
\hline Prostate cancer $^{\#}$ & 5 & $80.4 \pm 5.9$ & 5 & 1 & 1 & 0 & 0 & 0 & $1{ }^{\#}(20.0 \%)$ \\
\hline Biliary tract cancer $^{*}$ & 7 & $79.3 \pm 6.4$ & 3 & 1 & 0 & 0 & 0 & 0 & $1(14.3 \%)$ \\
\hline Lung cancer $^{+}$ & 6 & $70.3 \pm 11.0$ & 6 & 0 & 0 & 0 & 0 & 0 & $1+(16.7 \%)$ \\
\hline Pancreatic cancer & 10 & $67.6 \pm 10.3$ & 7 & 1 & 1 & 0 & 0 & 0 & $2(20.0 \%)$ \\
\hline Esophageal cancer & 4 & $65.8 \pm 10.7$ & 4 & 0 & 1 & 0 & 0 & 0 & $0(0 \%)$ \\
\hline Malignant lymphoma & 14 & $77.4 \pm 6.0$ & 8 & 2 & 1 & 2 & 0 & 0 & $1(7.1 \%)$ \\
\hline Myelodysplastic syndrome & 10 & $78.2 \pm 11.3$ & 4 & 1 & 0 & 0 & 1 & 0 & $0(0 \%)$ \\
\hline Others & 5 & $67.7 \pm 9.4$ & 2 & 2 & 0 & 1 & 0 & 0 & $0(0 \%)$ \\
\hline Total & 96 & $73.9 \pm 9.3$ & 68 & 12 & 7 & 4 & 2 & 1 & $9(9.4 \%)$ \\
\hline
\end{tabular}

*, a patient with hepatocellular carcinoma and colon cancer; ${ }^{*}$, a patient with stomach and prostate cancer; ${ }^{*}$, a patient with stomach and biliary tract cancer; ${ }^{+}$, a patient with stomach and lung cancer; PAVTE, peripheral arterial and venous thromboembolism, ACI, acute cerebral infarction; ACS, acute coronary syndrome; DIC, disseminated intravascular coagulation; TMA, thrombotic microangiopathic anemia.

Embolisms $(n=2)$ and peripheral artery thrombosis $(n=1)$. Nine patients $(9.4 \%)$ died within 28 days after the CWA examination.

Table 2 shows the peak time and height of the CWA-APTT in patients with various malignant neoplasms. The three peak times of the CWA-APTT were significantly longer in patients with stomach cancer, biliary tract cancer, pancreatic cancer, esophageal cancer, times of the STF/FIXa among patients with various malignant neoplasms and healthy volunteers. In contrast, the peak heights of the CWA-sTF/FIXa in patients with hepatocellular carcinoma, colon cancer, stomach cancer, prostate cancer, lung cancer, pancreatic cancer, esophageal cancer, and malignant lymphoma were significantly higher in comparison to those in healthy volunteers (Table 3).

Table 2. CWA-APTT in patients with malignant neoplasm.

\begin{tabular}{|c|c|c|c|c|c|c|}
\hline & 2nd DPT & 1st DPT & FFT & 2nd DPH & 1st DPH & FFH \\
\hline Cancer Type & & Seconds & & \multicolumn{3}{|c|}{ mm Absorbance } \\
\hline Hepatocellular carcinoma & $\begin{array}{c}32.7 \\
(26.8-42.0) \\
\end{array}$ & $\begin{array}{c}35.2 \\
(28.9-46.1) \\
\end{array}$ & $\begin{array}{c}38.2 \\
(31.1-53.4) \\
\end{array}$ & $\begin{array}{c}883^{* *} \\
(264-1981) \\
\end{array}$ & $\begin{array}{c}283 * \\
(139-801)\end{array}$ & $\begin{array}{c}250 * * * \\
(164-759)\end{array}$ \\
\hline Colon cancer & $\begin{array}{c}33.5 \\
(31.2-39.3)\end{array}$ & $\begin{array}{c}36.7 \\
(33.8-42.7)\end{array}$ & $\begin{array}{c}40.4 \\
(36.2-45.9)\end{array}$ & $\begin{array}{c}1235 * * \\
(1024-1452)\end{array}$ & $\begin{array}{c}460 \text { ** } \\
(330-509)\end{array}$ & $\begin{array}{c}387 * * \\
(286-447)\end{array}$ \\
\hline Stomach cancer & $\begin{array}{c}34.7 \\
(28.7-38.5)\end{array}$ & $\begin{array}{c}37.5 \\
(31.5-42.4)\end{array}$ & $\begin{array}{c}40.0 \\
(34.0-49.5) \text { * }\end{array}$ & $\begin{array}{c}773 \\
(450-1278)\end{array}$ & $\begin{array}{c}307 * \\
(147-457)\end{array}$ & $\begin{array}{c}279 * * * \\
(172-480)\end{array}$ \\
\hline Prostate cancer & $\begin{array}{c}34.8 \\
(29.5-60.7)\end{array}$ & $\begin{array}{c}37.7 \\
(32.2-65.4)\end{array}$ & $\begin{array}{c}39.9 \\
(34.4-67.8) \\
\end{array}$ & $\begin{array}{c}810 \\
(647-1220) \\
\end{array}$ & $\begin{array}{c}317 * * \\
(254-427)\end{array}$ & $\begin{array}{c}296 * * \\
(214-330)\end{array}$ \\
\hline Biliary tract cancer & $\begin{array}{c}34.0 * \\
(25.7-48.3)\end{array}$ & $\begin{array}{c}37.0 * \\
(28.0-58.8)\end{array}$ & $\begin{array}{c}40.6^{*} \\
(30.5-62.7)\end{array}$ & $\begin{array}{c}784 \\
(151-1789)\end{array}$ & $\begin{array}{c}315 \\
(90.7-544)\end{array}$ & $\begin{array}{c}257^{* * *} \\
(221-621)\end{array}$ \\
\hline
\end{tabular}


Table 2. Cont.

\begin{tabular}{|c|c|c|c|c|c|c|}
\hline & 2nd DPT & 1st DPT & FFT & 2nd DPH & 1st DPH & FFH \\
\hline Cancer Type & & Seconds & & \multicolumn{3}{|c|}{ mm Absorbance } \\
\hline Lung cancer & $\begin{array}{c}34.0 \\
(29.9-40.3)\end{array}$ & $\begin{array}{c}36.6 \\
(32.3-45.1) \\
\end{array}$ & $\begin{array}{c}39.5 \\
(34.2-49.5) \\
\end{array}$ & $\begin{array}{c}752 \\
(443-1004)\end{array}$ & $\begin{array}{c}259 \\
(132-450) \\
\end{array}$ & $\begin{array}{c}224 * \\
(178-480)\end{array}$ \\
\hline Pancreatic cancer & $\begin{array}{c}35.2^{* * *} \\
(32.6-39.3)\end{array}$ & $\begin{array}{c}38.5^{* * *} \\
(36.2-42.8)\end{array}$ & $\begin{array}{c}41.4^{* * *} \\
(38.1-48.2)\end{array}$ & $\begin{array}{c}973 * \\
(302-1422)\end{array}$ & $\begin{array}{c}353^{* *} \\
(254-559)\end{array}$ & $\begin{array}{c}336 * * * \\
(172-489)\end{array}$ \\
\hline Esophageal cancer & $\begin{array}{c}37.9 \\
(31.4-44.7)\end{array}$ & $\begin{array}{c}42.6 \\
(34.1-50.0)\end{array}$ & $\begin{array}{c}44.8 * \\
(36.9-51.9)\end{array}$ & $\begin{array}{c}1215^{* *} \\
(972-1572)\end{array}$ & $\begin{array}{c}491^{* *} \\
(418-595)\end{array}$ & $\begin{array}{c}448 * * \\
(317-477)\end{array}$ \\
\hline malignant lymphoma & $\begin{array}{c}36.1 * \\
(28.0-56.2)\end{array}$ & $\begin{array}{c}39.7^{*} \\
(30.5-60.5)\end{array}$ & $\begin{array}{c}43.9^{* *} \\
(32.5-68.7)\end{array}$ & $\begin{array}{c}863 \\
(274-1361)\end{array}$ & $\begin{array}{c}311^{* *} \\
(157-577)\end{array}$ & $\begin{array}{c}323 * * * \\
(195-634)\end{array}$ \\
\hline Myelodysplastic syndrome & $\begin{array}{c}37.5^{* * *} \\
(31.5-78.5)\end{array}$ & $\begin{array}{c}40.0 * * \\
(34.1-88.5)\end{array}$ & $\begin{array}{c}46.9^{* *} \\
(35.8-90.3)\end{array}$ & $\begin{array}{c}712 \\
(128-1144)\end{array}$ & $\begin{array}{c}337 \\
(157-577) \\
\end{array}$ & $\begin{array}{c}256^{* * *} \\
(182-653)\end{array}$ \\
\hline Others & $\begin{array}{c}38.1 \\
(31.1-44.5)\end{array}$ & $\begin{array}{c}43.3 * \\
(33.8-52.1)\end{array}$ & $\begin{array}{c}44.9 * \\
(35.8-55.6)\end{array}$ & $\begin{array}{c}845 \\
(596-1246)\end{array}$ & $\begin{array}{c}328 \\
(187-552)\end{array}$ & $\begin{array}{c}319 \\
(163-738)\end{array}$ \\
\hline Healthy volunteers & $\begin{array}{c}31.7 \\
(29.6-35.7)\end{array}$ & $\begin{array}{c}34.3 \\
(32.1-39.7)\end{array}$ & $\begin{array}{c}36.0 \\
(34.0-41.1)\end{array}$ & $\begin{array}{c}734 \\
(453-975)\end{array}$ & $\begin{array}{c}235 \\
(137-316)\end{array}$ & $\begin{array}{c}187 \\
(131-256)\end{array}$ \\
\hline
\end{tabular}

Data are shown as the median (range). CWA-APTT, clot waveform analysis-activated partial thromboplastin time; DPT, derivative peak time; DPH, derivative peak height; FFT, fibrin formation time; FFH, fibrin formation height; ${ }^{*}, p<0.05 ;{ }^{* *}, p<0.01 ; * * *, p<0.001$ compared to healthy volunteers.

Table 3. CWA-sTF/FIXa in patients with malignant neoplasm.

\begin{tabular}{|c|c|c|c|c|c|c|}
\hline & 2nd DPT & 1st DPT & FFT & 2nd DPH & 1st DPH & FFH \\
\hline Cancer Type & & Seconds & & \multicolumn{3}{|c|}{ mm Absorbance } \\
\hline Hepatocellular carcinoma & $\begin{array}{c}77.9 \\
(52.2-105)\end{array}$ & $\begin{array}{c}95.6 \\
(76.4-130)\end{array}$ & $\begin{array}{c}95.9 \\
(78.2-127)\end{array}$ & $\begin{array}{c}48.0 \\
(21.0-108)\end{array}$ & $\begin{array}{c}97.9^{* * *} \\
(49.9-270)\end{array}$ & $\begin{array}{c}352 \\
(205-938)\end{array}$ \\
\hline Colon cancer & $\begin{array}{c}83.2 \\
(76.6-97.6) \\
\end{array}$ & $\begin{array}{c}101.5 \\
(92.8-133)\end{array}$ & $\begin{array}{c}102 \\
(87.2-134)\end{array}$ & $\begin{array}{c}68.9 \\
(28.6-111)\end{array}$ & $\begin{array}{c}139 * * \\
(110-193)\end{array}$ & $\begin{array}{c}543 * \\
(334-639)\end{array}$ \\
\hline Stomach cancer & $\begin{array}{c}82.2 \\
(63.9-148)\end{array}$ & $\begin{array}{c}97.0 \\
(81.9-207)\end{array}$ & $\begin{array}{c}97.2 \\
(82.4-196)\end{array}$ & $\begin{array}{c}46.3 \\
(11.4-102)\end{array}$ & $\begin{array}{c}106^{*} \\
(28.3-178)\end{array}$ & $\begin{array}{c}370 \\
(156-564)\end{array}$ \\
\hline Prostate cancer & $\begin{array}{c}72.7 \\
(38.6-93.9)\end{array}$ & $\begin{array}{c}91.8 \\
(62.3-105)\end{array}$ & $\begin{array}{c}89.6 \\
(65.6-93.9)\end{array}$ & $\begin{array}{c}46.4 \\
(36.9-95.0)\end{array}$ & $\begin{array}{c}80.9 * \\
(64.8-178)\end{array}$ & $\begin{array}{c}455 \\
(257-934)\end{array}$ \\
\hline Biliary tract cancer & $\begin{array}{c}83.0 \\
(46.3-148)\end{array}$ & $\begin{array}{c}103 \\
(79.3-207)\end{array}$ & $\begin{array}{c}102 \\
(83.1-196)\end{array}$ & $\begin{array}{c}70.5 \\
(13.8-109)\end{array}$ & $\begin{array}{c}111 \\
(28.3-250)\end{array}$ & $\begin{array}{c}364 \\
(184-702)\end{array}$ \\
\hline Lung cancer & $\begin{array}{c}72.6 \\
(66.6-115)\end{array}$ & $\begin{array}{c}82.6 \\
(76.4-146)\end{array}$ & $\begin{array}{c}84.9 \\
(78.0-152)\end{array}$ & $\begin{array}{c}49.1 \\
(26.6-84.1)\end{array}$ & $\begin{array}{c}92.0 \\
(46.1-181) *\end{array}$ & $\begin{array}{c}314 \\
(183-645)\end{array}$ \\
\hline Pancreatic cancer & $\begin{array}{c}80.9 \\
(70.6-102)\end{array}$ & $\begin{array}{c}102 \\
(91.2-133)\end{array}$ & $\begin{array}{c}103 \\
(88.7-132)\end{array}$ & $\begin{array}{c}36.0 \\
(19.0-72.6)\end{array}$ & $\begin{array}{c}121 \\
(46.2-165) * *\end{array}$ & $\begin{array}{c}411 \\
(163-602) *\end{array}$ \\
\hline Esophageal cancer & $\begin{array}{c}74.8 \\
(73.6-85.0) \\
\end{array}$ & $\begin{array}{c}98.7 \\
(90.9-113) \\
\end{array}$ & $\begin{array}{c}98.9 \\
(89.9-115)\end{array}$ & $\begin{array}{c}69.8 \\
(43.5-112) *\end{array}$ & $\begin{array}{c}173 \\
(123-213)^{* *}\end{array}$ & $\begin{array}{c}600 \\
(464-645) * * \\
\end{array}$ \\
\hline malignant lymphoma & $\begin{array}{c}76.1 \\
(50.0-147)\end{array}$ & $\begin{array}{c}93.1 \\
(72.0-178)\end{array}$ & $\begin{array}{c}92.0 \\
(74.9-174)\end{array}$ & $\begin{array}{c}45.6 \\
(17.3-173)\end{array}$ & $\begin{array}{c}101 \\
(62.5-304) * * *\end{array}$ & $\begin{array}{c}437 \\
(246-751)^{* *}\end{array}$ \\
\hline Myelodysplastic syndrome & $\begin{array}{c}90.5 \\
(52.2-189) \\
\end{array}$ & $\begin{array}{c}113 \\
(60.2-237) \\
\end{array}$ & $\begin{array}{c}111 \\
(61.2-225) \\
\end{array}$ & $\begin{array}{c}58.6 \\
(9.8-69.8) \\
\end{array}$ & $\begin{array}{c}91.4 \\
(33.6-222) \\
\end{array}$ & $\begin{array}{c}329 \\
(265-734) \\
\end{array}$ \\
\hline Others & $\begin{array}{c}106 \\
(73.6-132) *\end{array}$ & $\begin{array}{c}129 \\
(84.8-171)\end{array}$ & $\begin{array}{c}126 \\
(84.2-169)\end{array}$ & $\begin{array}{c}38.9 \\
(15.1-47.7)\end{array}$ & $\begin{array}{c}69.6 \\
(36.4-189)\end{array}$ & $\begin{array}{c}406 \\
(285-832)\end{array}$ \\
\hline Healthy volunteers & $\begin{array}{c}76.9 \\
(62.5-106)\end{array}$ & $\begin{array}{c}95.3 \\
(76.7-140)\end{array}$ & $\begin{array}{c}94.6 \\
(80.3-139)\end{array}$ & $\begin{array}{c}37.4 \\
(21.2-60.9)\end{array}$ & $\begin{array}{c}67.8 \\
(37.2-88.3)\end{array}$ & $\begin{array}{c}341 \\
(221-495)\end{array}$ \\
\hline
\end{tabular}

Data are shown as the median (range). CWA-sTF/FIXa, clot waveform analysis-small amount tissue factor induced FIX activation assay; DPT, derivative peak time; DPH, derivative peak height; FFT, fibrin formation time; FFH, fibrin formation height; ${ }^{*}, p<0.05 ;{ }^{* *}, p<0.01 ;{ }^{* * *}$, $p<0.001$ compared to healthy volunteers. 
Regarding the relationship between the CWA and thrombosis, patients treated with anticoagulants were excluded. There were no significant differences in the three peak times of the CWA-APTT among healthy volunteers, and malignant neoplasm patients with or without thrombosis (Figure 2a). The three peak heights of the CWA-APTT were significantly higher in malignant neoplasm patients with or without thrombosis than in healthy volunteers. FFH was the only parameter that was significantly higher in those with thrombosis than in those without (Figure 2b). The three peak times of the CWA-sTF/FIXa were significantly longer in malignant neoplasm patients with or without thrombosis in comparison to healthy volunteers (Figure 3a). The three peak heights of the CWA-sTF/FIXa were significantly higher in those patients with or without thrombosis than in healthy volunteers. The 2nd DPH and 1st DPH of the CWA-sTF/FIXa were significantly higher in those patients with thrombosis than in those without thrombosis (Figure 3b).

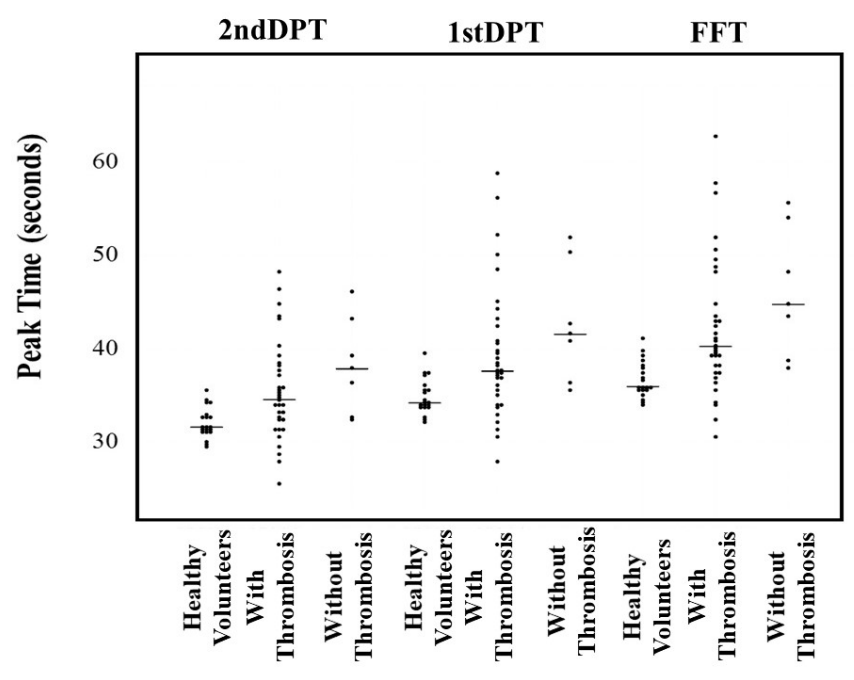

(a)

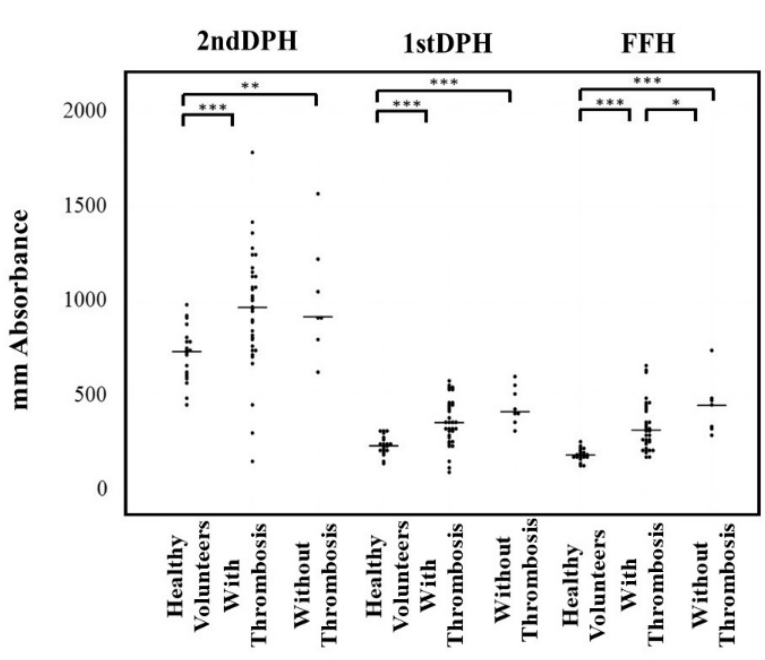

(b)

Figure 2. Peak time (a) and height (b) of activated partial thromboplastin time. DPT, derivative peak time; FFT, fibrin formation time; DPH, derivative peak height; FFH, fibrin formation height; ${ }^{*}, p<0.05 ;{ }^{* *}, p<0.01 ;{ }^{* * *}, p<0.001$.

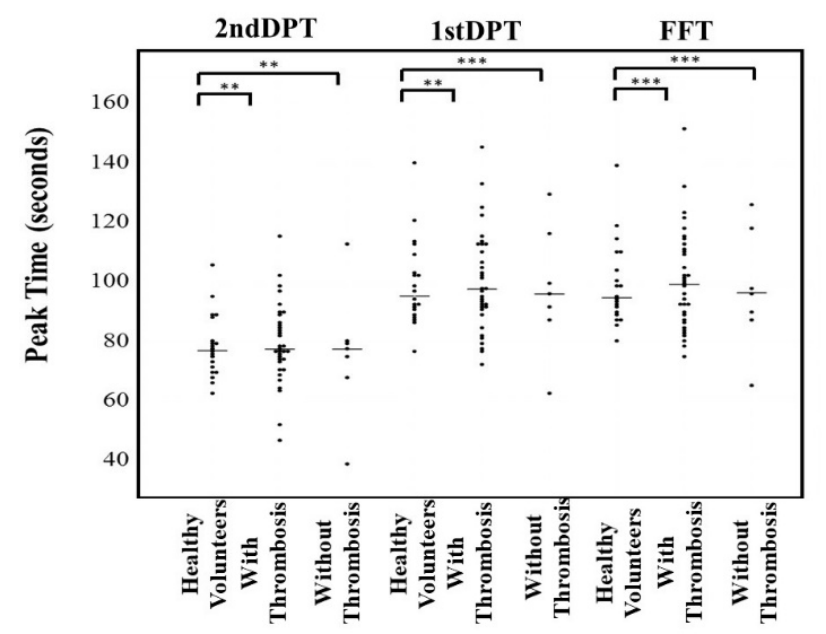

(a)

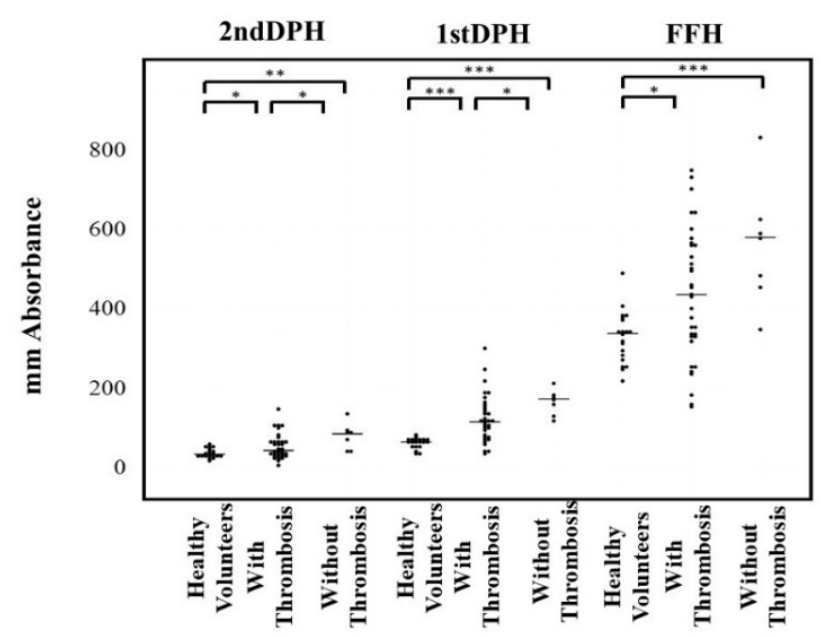

(b)

Figure 3. Peak time (a) and height (b) of small amount of tissue factor induced FIX activation assay. DPT, derivative peak time; FFT, fibrin formation time; DPH, derivative peak height; FFH, fibrin formation height; ${ }^{*}, p<0.05 ;{ }^{* *}, p<0.01$; $* * *, p<0.001$. 


\section{Discussion}

In this study, $28.3 \%$ of patients with malignant neoplasm had thrombotic diseases, suggesting that these patients were in a hypercoagulable state. Patients with malignant neoplasm are frequently associated with thrombotic disorder and are considered to be in a hypercoagulable state [21]. Trousseaus syndrome has been shown in multiple published articles [22-26] to have a relationship between malignant neoplasms and thrombotic disease [6]. The existence of multiple definitions of Trousseau's syndrome, or cancer- associated thrombosis, is due in part to the existence of multiple pathophysiologic mechanisms that apparently contribute to the hypercoagulability associated with cancer [6]. The mechanism underlying cancer-associated thrombosis reportedly involves the over-expression of TF [22], cysteine proteinase [23], tumor hypoxia [24], carcinomas mucins [25], and oncogene activation [26]. These risk factors overlap to cause thrombosis in patients with cancer [6]. Although the specific relationship between the type of neoplasm and thrombotic diseases is not clear due to the small size of this study, cases of stomach cancer and hematological malignancy with DIC [27,28], hepatocellular carcinoma with portal vein thrombosis [29,30], and pancreatic cancer and brain tumor with venous thromboembolism [31] have been previously reported. As cancer-associated thrombosis causes a fatal hypercoagulable state, anticoagulation therapy should be administered to these patients. Therefore, the early detection of a hypercoagulable state may be important.

Routine assays as APTT and prothrombin time are generally used for monitoring anticoagulant or diagnosing bleeding disorders, but they are not used to evaluate the hypercoagulability. Elevated D-dimer levels are useful to suspect venous thromboembolism [32] but cannot evaluate the hypercoagulability. Although thrombin generation test or thromboelastography may detect the hypercoagulability, these tests are not routine assay. The peak height of 1st and 2nd DP of APTT was reported to able to evaluate hemostatic ability [16]. The present study showed that the peak times of APTT which correspond routine APTT, could not evaluate the hypercoagulability in patients with malignant neoplasm. Although the peak height of APTT was significantly high in patients with malignant neoplasm, there were no significant difference in the peak height of APTT between these patients with and without thrombosis. Therefore, APTT included the activator for contact factors and massive phospholipid, suggesting that APTT cannot show a physiological coagulation ability $[16,18]$.

The CWA-sTF/FIXa assay uses plalet-rich plasma as physiological phospholipid which does not activate contact factor and reflects platelet activation $[16,18]$. The prolongation of peak times in patients with malignant neoplasm reflect with anticoagulants. The prolongation of peak time in CWA-APTT and STF/FIXa due to anticoagulant were previously reported in major orthopedic surgery [17]. The peak time CWA-APTT or -sTF/FIXa may reflect with anticoagulant, and the peak height may reflect physiological clotting activity. The peak heights of CWA-sTF/FIXa show not only hypercoagulability in patients with malignant neoplasm but also possibility of prediction of thrombosis. One case (Figure 1(I-c,II-c)) showed markedly elevated peak height before onset of acute cerebral infarction. This case should be treated with anticoagulant.

\section{Conclusions}

Patients with malignant neoplasm are in a hypercoagulable state and often associated with thrombosis. The peak height of CWA-APTT and sTF/FIXa was extremely high in patients with malignant neoplasm, especially those with thrombosis, suggesting that the peak height of CWA-sTF/FIXa may be useful to detect the hypercoagulability.

Author Contributions: Conceptualization, H.W. and K.S.; methodology, Y.I.; software, H.W.; validation, H.I. and K.S.; formal analysis, H.W.; investigation, I.M.; data curation, S.F.; writing-original draft preparation, M.K.; writing—review and editing, H.W.; visualization, H.M.; supervision, H.S.; project administration, M.S.; funding acquisition, H.W. All authors have read and agreed to the published version of the manuscript. 
Funding: This research was funded by a grant-in-aid from the Ministry of Health, Labour and Welfare of Japan (H30-105).

Institutional Review Board Statement: The study protocol (O-0057) was approved by the Human Ethics Review committees of Mie Prefectural General Medical Center, and informed consent was obtained from each patient.

Informed Consent Statement: Informed consent was obtained from all subjects involved in the study.

Data Availability Statement: The data presented in this study are available on request to the corresponding author. The data are not publicly available due to privacy restrictions.

Acknowledgments: The authors thank Nisii $\mathrm{H}$ and Sakano $\mathrm{Y}$ for their kind support in performing the assay for the CWA. The authors also thank the many technicians at Central Laboratory for measuring the laboratory data and the many physicians who took care of the patients.

Conflicts of Interest: The measurements of CWA were partially supported by Instrumentation Laboratory Japan. In the other points, the authors declare no conflicts of interest.

\section{References}

1. Hisada, Y.; Mackman, N. Cancer-associated pathways and biomarkers of venous thrombosis. Blood 2017, 130, 1499-1506. [CrossRef]

2. Mahajan, A.; Brunson, A.; White, R.; Wun, T. The Epidemiology of Cancer-Associated Venous Thromboembolism: An Update. Semin. Thromb. Hemost. 2019, 45, 321-325. [CrossRef]

3. Fernandes, C.J.; Morinaga, L.T.K.; Alves, J.L., Jr.; Castro, M.A.; Calderaro, D.; Jardim, C.V.P.; Souza, R. Cancer-associated thrombosis: The when, how and why. Eur. Respir. Rev. 2019, 28, 180119. [CrossRef]

4. Kim, A.S.; Khorana, A.A.; McCrae, K.R. Mechanisms and biomarkers of cancer-associated thrombosis. Transl. Res. 2020, 225, 33-53. [CrossRef]

5. Timp, J.F.; Braekkan, S.K.; Versteeg, H.H.; Cannegieter, S.C. Epidemiology of cancer-associated venous thrombosis. Blood 2013, 122, 1712-1723. [CrossRef]

6. Varki, A. Trousseau's syndrome: Multiple definitions and multiple mechanisms. Blood 2007, 110, 1723-1729. [CrossRef]

7. Trousseau, A. Plegmasia alba dolens. In Lectures on Clinical Medicine; Hotel-Dieu: Paris, France, 1865; Volume 5, pp. $281-332$.

8. Sack, G.H.; Levin, J.; Bell, W.R. Trousseau's syndrome and other manifestations of chronic disseminated coagulopathy in patients with neoplasms: Clinical, pathophysiologic, and therapeutic features. Medicine 1977, 56, 1-37. [CrossRef] [PubMed]

9. Bao, L.; Zhang, S.; Gong, X.; Cui, G. Trousseau Syndrome Related Cerebral Infarction: Clinical Manifestations, Laboratory Findings and Radiological Features. J. Stroke Cereb. Dis. 2020, 29, 104891. [CrossRef] [PubMed]

10. Winter, P.C. The pathogenesis of venous thromboembolism in cancer: Emerging links with tumour biology. Hematol. Oncol. 2006, 24, 126-133. [CrossRef] [PubMed]

11. Khorana, A.A. Malignancy, thrombosis and Trousseau: The case for an eponym. J. Thromb. Haemost. 2003, 1, 2463-2465. [CrossRef]

12. Sharma, B.K.; Flick, M.J.; Palumbo, J.S. Cancer-Associated Thrombosis: A Two-Way Street. Semin. Thromb. Hemost. 2019, 45, 559-568. [CrossRef] [PubMed]

13. Suzuki-Inoue, K. Platelets and cancer-associated thrombosis: Focusing on the platelet activation receptor CLEC-2 and podoplanin. Blood 2019, 134, 1912-1918. [CrossRef]

14. Maestre, A.; Trujillo-Santos, J.; Visoná, A.; Lobo, J.L.; Grau, E.; Malý, R.; Duce, R.; Monreal, M.; RIETE Investigators. D-dimer levels and 90-day outcome in patients with acute pulmonary embolism with or without cancer. Thromb. Res. 2014, 133, 384-389. [CrossRef] [PubMed]

15. Gerotziafas, G.T.; Mahé, I.; Lefkou, E.; AboElnazar, E.; Abdel-Razeq, H.; Taher, A.; Antic, D.; Elalamy, I.; Syrigos, K.; Van Dreden, P. Overview of risk assessment models for venous thromboembolism in ambulatory patients with cancer. Thromb. Res. 2020, 191, S50-S57. [CrossRef]

16. Wada, H.; Matsumoto, T.; Ohishi, K.; Shiraki, K.; Shimaoka, M. Update on the Clot Waveform Analysis. Clin. Appl. Thromb. Hemost. 2020, 26, 1076029620912027. [CrossRef] [PubMed]

17. Hasegawa, M.; Tone, S.; Wada, H.; Naito, Y.; Matsumoto, T.; Yamashita, Y.; Shimaoka, M.; Sudo, A. The evaluation of hemostatic abnormalities using a CWA-small amount tissue factor induced FIX activation assay in major orthopedic surgery patients. Clin. Appl. Thromb. Hemost. 2021, 27, 1076029620976913. [CrossRef]

18. Wada, H.; Shiraki, K.; Matsumoto, T.; Ohishi, K.; Shimpo, H.; Shimaoka, M. Effects of platelet and phospholipids on clot formation activated by a small amount of tissue factor. Thromb. Res. 2020, 193, 146-153. [CrossRef]

19. Kobayashi, N.; Maegawa, T.; Takada, M.; Tanaka, H.; Gonmori, H. Criteria for diagnosis of DIC based on the analysis of clinical and laboratory findings in 345 DIC patients collected by the Research Committee on DIC in Japan. Bibl. Haemotol. 1983, 49, 265-275.

20. Matsumoto, T.; Wada, H.; Fujimoto, N.; Toyoda, J.; Abe, Y.; Ohishi, K.; Yamashita, Y.; Ikejiri, M.; Hasegawa, K.; Suzuki, K.; et al. An Evaluation of the Activated Partial Thromboplastin Time Waveform. Clin. Appl. Thromb. Hemost. 2018, 24, 764-770. [CrossRef] 
21. Falanga, A.; Marchetti, M.; Russo, L. Hemostatic Biomarkers and cancer prognosis: Where Do We Stand? Semin. Thromb. Hemost. 2021, Online ahead of print. [CrossRef]

22. Rak, J.; Milsom, C.; May, L.; Klement, P.; Yu, J. Tissue factor in cancer and angiogenesis: The molecular link between genetic tumor progression, tumor neovascularization, and cancer coagulopathy. Semin. Thromb. Hemost. 2006, 32, 54-70. [CrossRef]

23. Gordon, S.G.; Cross, B.A. A factor X-activating cysteine protease from malignant tissue. J. Clin. Investig. 1981, 67, 1665-1671. [CrossRef] [PubMed]

24. Denko, N.C.; Giaccia, A.J. Tumor hypoxia, the physiological link between Trousseau's syndrome (carcinoma-induced coagulopathy) and metastasis. Cancer Res. 2001, 61, 795-798. [PubMed]

25. Wahrenbrock, M.; Borsig, L.; Le, D.; Varki, N.; Varki, A. Selectin-mucin interactions as a probable molecular explanation for the association of Trousseau syndrome with mucinous adenocarcinomas. J. Clin. Investig. 2003, 112, 853-862. [CrossRef] [PubMed]

26. Boccaccio, C.; Sabatino, G.; Medico, E.; Girolami, F.; Follenzi, A.; Reato, G.; Sottile, A.; Naldini, L.; Comoglio, P. The MET oncogene drives a genetic programme linking cancer to haemostasis. Nature 2005, 434, 396-400. [CrossRef]

27. Hwang, I.G.; Choi, J.H.; Park, S.H.; Oh, S.Y.; Kwon, H.C.; Lee, S.I.; Lim, D.H.; Lee, G.W.; Kang, J.H. Chemotherapy in advanced gastric cancer patients associated with disseminated intravascular coagulation. Cancer Res. Treat. 2014, 46, 27-32. [CrossRef]

28. Wada, H.; Matsumoto, T.; Yamashita, Y.; Hatada, T. Disseminated Intravascular Coagulation: Testing and Diagnosis. Clin. Chim. Acta 2014, 436C, 130-134. [CrossRef]

29. Rimassa, L.; Personeni, N.; Czauderna, C.; Foerster, F.; Galle, P. Systemic treatment of HCC in special populations. J. Hepatol. 2021, 74, 931-943. [CrossRef]

30. Khan, A.R.; Wei, X.; Xu, X. Portal Vein Tumor Thrombosis and Hepatocellular Carcinoma-The Changing Tides. J. Hepatocell. Carcinoma 2021, 8, 1089-1115. [CrossRef]

31. White, R.H.; Chew, H.K.; Zhou, H.; Parikh-Patel, A.; Harris, D.; Harvey, D.; Wun, T. Incidence of venous thromboembolism in the year before the diagnosis of cancer in 528,693 adults. Arch. Intern. Med. 2005, 165, 1782-1787. [CrossRef]

32. Johnson, E.D.; Schell, J.C.; Rodgers, G.M. The D-dimer assay. Am. J. Hematol. 2019, 94, 833-839. [CrossRef] [PubMed] 